P0107

\title{
REFERENCE RADIOMETER IN A DUAL-PHOTODIODE DESIGN FOR CALIBRATION OF UVA IRRADAINCE METERS
}

\author{
Dong-Joo Shin et al.
}

DOI 10.25039/x46.2019.PO107

from

CIE x046:2019

Proceedings

of the

29th CIE SESSION

Washington D.C., USA, June 14 - 22, 2019

(DOI 10.25039/x46.2019)

The paper has been presented at the 29th CIE Session, Washington D.C., USA, June 14-22, 2019. It has not been peer-reviewed by CIE.

(C) CIE 2019

All rights reserved. Unless otherwise specified, no part of this publication may be reproduced or utilized in any form or by any means, electronic or mechanical, including photocopying and microfilm, without permission in writing from CIE Central Bureau at the address below. Any mention of organizations or products does not imply endorsement by the CIE.

This paper is made available open access for individual use. However, in all other cases all rights are reserved unless explicit permission is sought from and given by the CIE.

CIE Central Bureau

Babenbergerstrasse 9

A-1010 Vienna

Austria

Tel.: +4317143187

e-mail: ciecb@cie.co.at

www.cie.co.at 


\title{
REFERENCE RADIOMETER IN A DUAL-PHOTODIODE DESIGN FOR CALIBRATION OF UVA IRRADAINCE METERS
}

\author{
Shin, D.-J., Park, S., Jeong, K., Lee, D.-H. \\ Korea Research Institute of Standards and Science, Daejeon, REPUBLIC OF KOREA \\ djshin@kriss.re.kr
}

DOI 10.25039/x46.2019.PO107

\begin{abstract}
We present a new design of a reference radiometer for calibration of UVA irradiance meters, which can be used for any source spectrum as long as its wavelength range is limited from 330 $\mathrm{nm}$ to $450 \mathrm{~m}$. It is based on the concept of dual-photodiode radiometer, which can simultaneously measure both the centroid wavelength and the spectrally integrated irradiance of sources with a finite spectral bandwidth. The realization of the concept in the UVA range is presented with the experimentally measured characteristics. The results measured with the dual-photodiode radiometer are compared with a spectroradiometer and an absolute pyroelectric radiometer for the centroid wavelength and irradiance measurements, respectively, at various UV source: a diode laser at $404 \mathrm{~nm}$, a high pressure $\mathrm{Hg}$ lamp filtered at $365 \mathrm{~nm}$ and $405 \mathrm{~nm}$ with a bandwidth of approximately $15 \mathrm{~nm}$, and two UV LEDs with peak wavelengths at $365 \mathrm{~nm}$ and $385 \mathrm{~nm}$. We confirmed the validity of the new radiometer with an agreement of less than $0.2 \mathrm{~nm}$ and $0.5 \%$ for the centroid wavelength and irradiance, respectively.
\end{abstract}

Keywords: UVA irradiance meter, dual-photodiode radiometer, centroid wavelength, spectrally integrated irradiance, spectrally flat responsivity

\section{Introduction}

UVA irradiance meters (UVA-meters) measure the spectrally integrated irradiance of UV sources in a finite spectral bandwidth with a peak at $365 \mathrm{~nm}$. One of the calibration method for UVA-meters recommended by CIE [CIE, 2016] is the comparison against a reference radiometer calibrated at a single spectral line. In this method, a filtered narrow-band mercury line source is often used. When the reference radiometer used for the calibration has a spectrally flat responsivity in the whole range for UVA meters, the calibration results can be valid for other sources. In this sense, a pyroelectric radiometer could be the desirable reference radiometer for calibration of UVA-meters [Eppeldauer, 2017]. In practice, however, a reference radiometer based on a silicon photodiode is widely used due to its advantages such as high sensitivity, fast response, and wide dynamic range. The shortcoming of the photodiode-based reference radiometer is that it undergoes a systematic error when the spectrum of the calibration source changes, which originates from the spectral dependence of its responsivity.

In this work, we present a new design of a reference radiometer for the UVA-meter calibration, which can be used for any source spectrum as long as its wavelength range is limited from 330 $\mathrm{nm}$ to $450 \mathrm{~nm}$. The new design is based on the dual-photodiode radiometer, which can simultaneously measure both the centroid wavelength and the spectrally integrated irradiance of light sources having finite spectral bandwidths [Shin, 2019]. We experimentally realized the dual-photodiode radiometer concept in the UV range and tested its validity by comparing with a spectroradiometer and an ECPR (Electrically Calibrated Pyroelectric Radiometer) at various UV sources: a diode laser at a wavelength of $404 \mathrm{~nm}$, a high pressure $\mathrm{Hg}$ lamp filtered at 365 $\mathrm{nm}$ and $405 \mathrm{~nm}$ with a bandwidth of approximately $15 \mathrm{~nm}$, and also UV LEDs at $365 \mathrm{~nm}$ and $385 \mathrm{~nm}$. For all the sources, the dual-photodiode radiometer showed an agreement of less than $0.2 \mathrm{~nm}$ with the spectroradiometer and less than $1 \%$ with the ECPR for the centroid wavelength and irradiance measurement, respectively. In conclusion, we expect that the dual-photodiode radiometer can replace an absolute radiometer with a spectrally flat responsivity or a spectroradiometer to realize the calibration of UVA-meters with various types of narrow band UV sources, including UV LEDs. 


\section{Reference radiometer realization}

\subsection{Setup}

Figure 1 shows the dual-photodiode radiometer setup. It consists of an aperture, a diffuser, a beam splitter, and two silicon photodiodes. A fused silica diffuser is used just behind the front aperture to reduce the alignment error when it is compared with other radiometers. A light trap was used to remove the UV radiation reflected from photodiode surfaces.

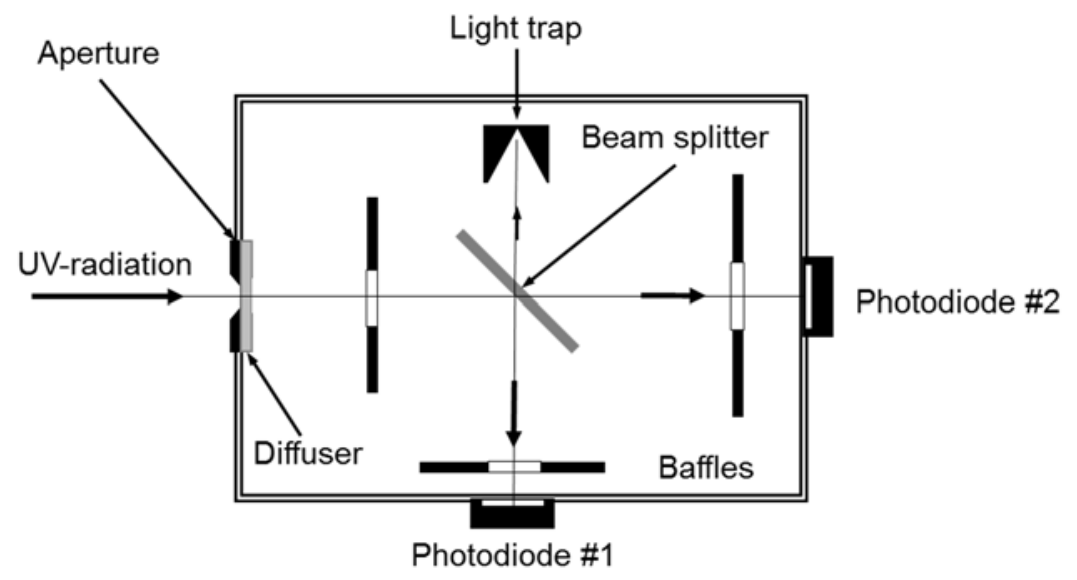

Figure 1 - Schematic setup of reference radiometer in a dual-photodiode design.

Spectral irradiance responsivities of two photodiodes were engineered to approximately be linear over the wavelength range from $330 \mathrm{~nm}$ to $450 \mathrm{~nm}$ by using a coloured filter (SCHOTT Glass \#: BG7) installed at an angle of $45^{\circ}$ as a beam splitter. The UV radiation incident on the diffuser is clipped by the front aperture, each photodiode measures the fraction of the radiation transmitted and reflected from the beam splitter, respectively. Figure 2 shows the spectral transmittance of the filter measured at the normal incidence condition.

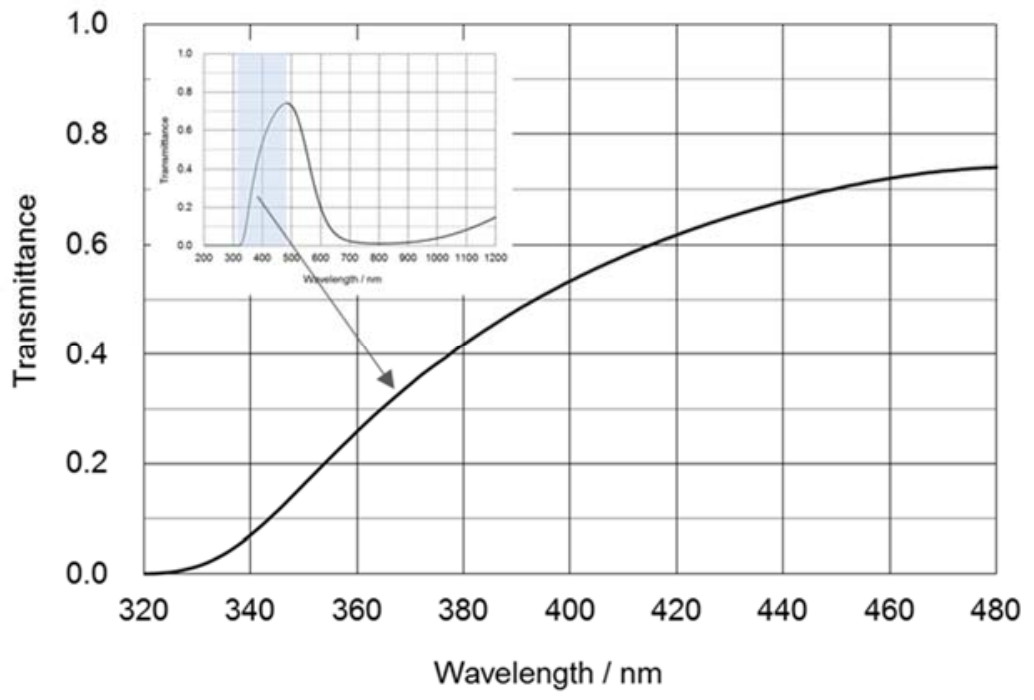

Figure 2 - Spectral transmittance of the filter (measured at normal incidence).

\subsection{Measurement equations}

The working principle of the dual-photodiode radiometer are described in details in another reference [Shin, 2019]. Here, the essential measurement equations are summarized.

Denoting $R_{1}(\lambda)$ and $R_{2}(\lambda)$ the spectral irradiance responsivities of two photodiodes \#1 and \#2, respectively, as shown in Figure 1, we define the ratio of two quantities as 


$$
r(\lambda)=\frac{R_{2}(\lambda)}{R_{1}(\lambda)}
$$

The dual-photodiode radiometer is regarded as calibrated when the responsivities $R_{1}(\lambda)$ and $R_{2}(\lambda)$ are measured and the ratio $r(\lambda)$ is calculated in its operating wavelength range. The dualphotodiode radiometer works only when the following conditions are fulfilled: first, the spectral responsivities $R_{1}(\lambda)$ and $R_{2}(\lambda)$ should be a linear function against wavelength within the spectral bandwidth of the light source to be measured. Second, the function $r(\lambda)$ should be a monotonic function against wavelength so that its reversed function $r^{-1}(x)$ can exist at any point within the operating wavelength range.

When the two conditions mentioned above are fulfilled, we can derive the following equation:

$$
\frac{i_{2}}{i_{1}}=\frac{R_{2}\left(\lambda_{c}\right)}{R_{1}\left(\lambda_{c}\right)}=r\left(\lambda_{c}\right),
$$

where $i_{1}$ and $i_{2}$ denote the photocurrent readings of photodiode \#1 and \#2, respectively, and $\lambda_{c}$ the centroid wavelength of the incident UV source defined as

$$
\lambda_{\mathrm{c}}=\frac{\int_{\lambda_{1}}^{\lambda_{2}} \lambda \cdot E_{\lambda}(\lambda) \mathrm{d} \lambda}{\int_{\lambda_{1}}^{\lambda_{2}} E_{\lambda}(\lambda) \mathrm{d} \lambda}
$$

for the spectral irradiance $E_{\lambda}(\lambda)$ with a finite spectral bandwidth from $\lambda_{1}$ to $\lambda_{2}$. Consequently, the measurement equation for the centroid wavelength is

$$
\lambda_{c}=r^{-1}\left(\frac{i_{2}}{i_{1}}\right) .(4)
$$

Once the centroid wavelength is determined, the spectrally integrated irradiance $E=$ $\int_{\lambda_{1}}^{\lambda_{2}} E_{\lambda}(\lambda) \mathrm{d} \lambda$ can be measured from the following equation:

$$
E=\frac{i_{1}}{R_{1}\left(\lambda_{c}\right)}\left(=\frac{i_{2}}{R_{2}\left(\lambda_{c}\right)}\right) .
$$

\subsection{Spectral irradiance responsivity calibration}

Spectral irradiance responsivities of the dual-photodiode reference radiometer in a range from $300 \mathrm{~nm}$ to $450 \mathrm{~nm}$ are measured in three steps: first, the relative spectral power responsivity is measured by using a lamp-based spectral responsivity comparator setup. Second, the absolute power responsivity is measured at one laser wavelength by using a calibrated photodiode. Third, the area of the precision aperture is measured by using a calibrated 3-D profiler to calculate the absolute irradiance responsivity.

Figure 3 shows the results of the calibration measurements for the spectral irradiance responsivities $R_{1}(\lambda)$ and $R_{2}(\lambda)$ and their ratio $r(\lambda)$. We see that the spectral responsivity $R_{1}(\lambda)$ is close to a linear function against wavelength in the whole operating range from $330 \mathrm{~nm}$ to $450 \mathrm{~nm}$, while the spectral responsivity $R_{2}(\lambda)$ shows a slowly varying deviation caused by the transmittance of the filter shown in Figure 2. In this case, we expect a bandwidth-dependent measurement error as shown from the numerical simulation in [Shin, 2019]. The ratio $r(\lambda)$ is a monotonic function in the whole operating range. 


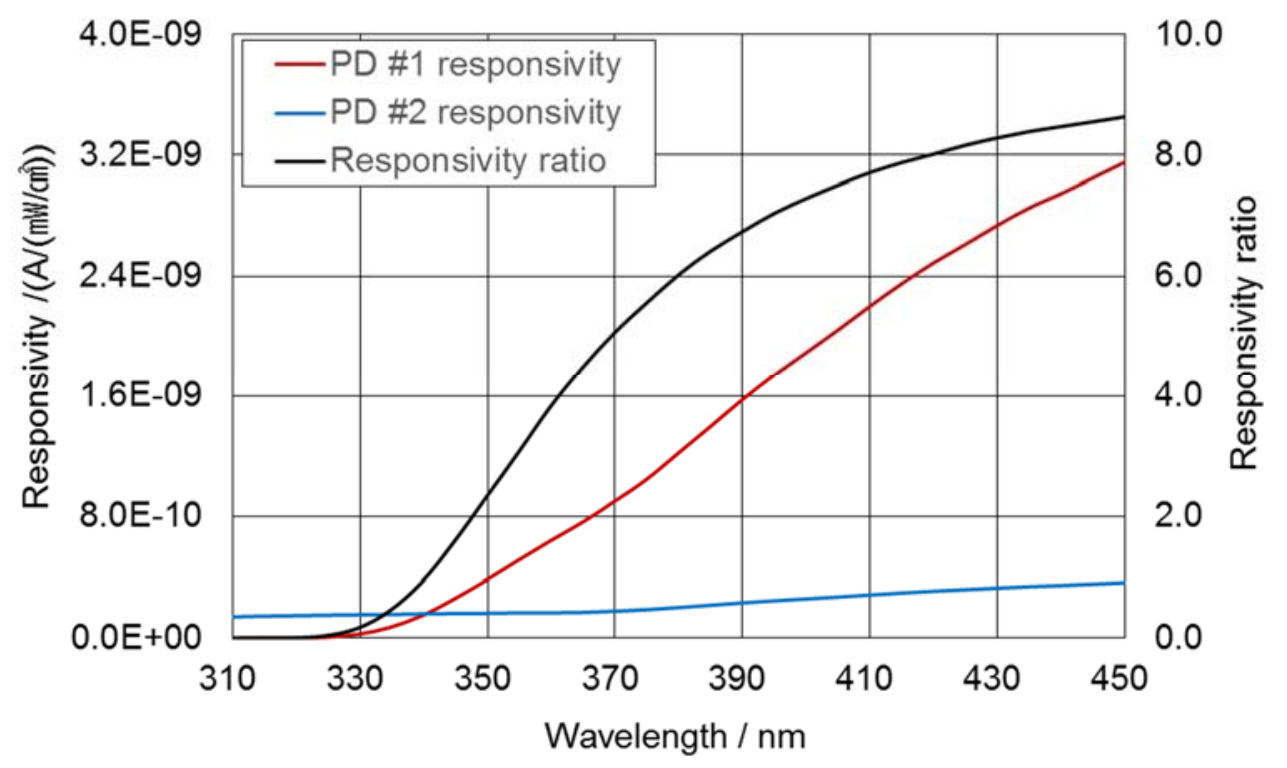

Figure 3 - Spectral irradiance responsivities of the dual-photodiode reference radiometer and their ratio.

\section{Experimental results}

For the validity test, the measurement results of centroid wavelength and irradiance by the dualphotodiode reference radiometer are compared with a spectroradiometer and an ECPR, respectively, at various finite-bandwidth UV sources: a diode laser at a wavelength of $404 \mathrm{~nm}$, a high pressure $\mathrm{Hg}$ lamp filtered at $365 \mathrm{~nm}$ and $405 \mathrm{~nm}$ with a bandwidth of approximately 15 $\mathrm{nm}$, and also UV LEDs with peak wavelengths at $365 \mathrm{~nm}$ and $385 \mathrm{~nm}$. Figure 4 shows the experimental setup for the comparison measurement.

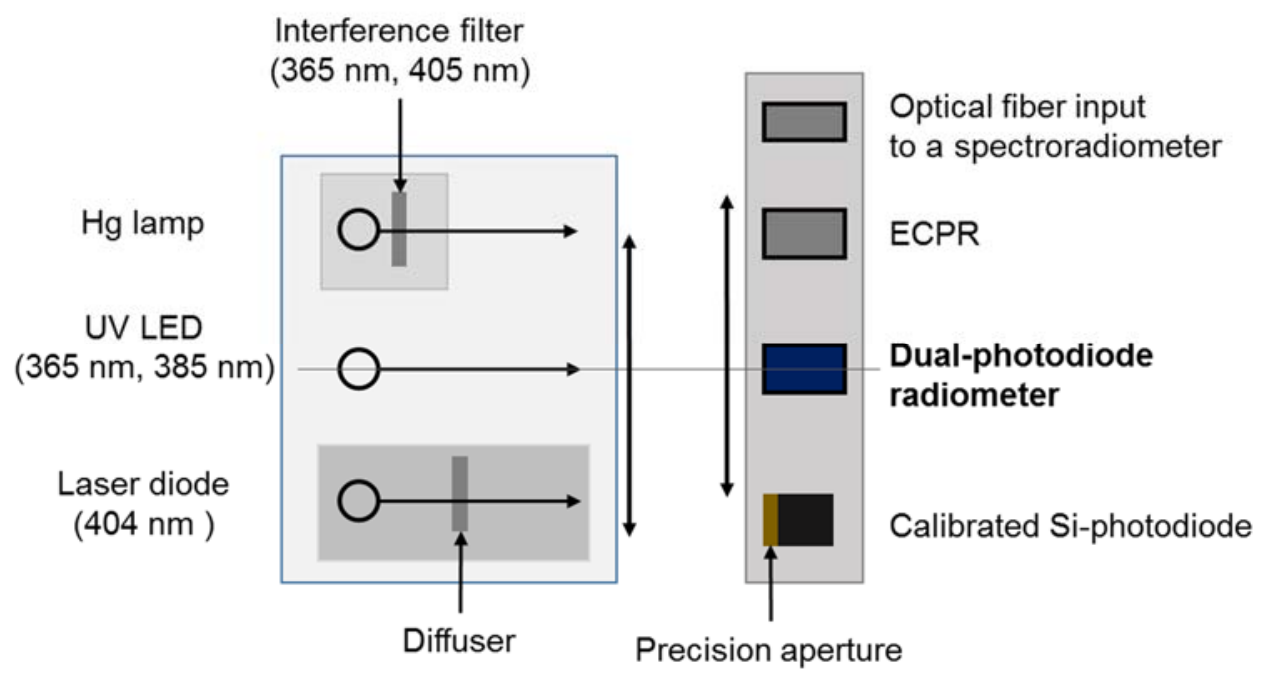

\section{Figure 4 - Experimental setup for comparison measurement of radiometers at various UV sources.}

The spectroradiometer with an optical fibre input is used to measure the relative spectral distribution of the source, from which the centroid wavelength can be calculated. The spectroradiometer is calibrated by using the spectral irradiance standard lamp of KRISS. The ECPR is the transfer standard for the UVA-meter calibration at KRISS, which provides the spectrally flat responsivity in a wide range from UV to IR. The absolute responsivity of the ECPR is calibrated traceable to the cryogenic radiometer of KRISS. The calibrated Si photodiode in 
Figure 4 with a precision aperture is used to calibrate the absolute irradiance responsivity of the dual-photodiode radiometer at the laser wavelength of $404 \mathrm{~nm}$.

Figure 5 shows the measured relative spectral distribution of the UV sources together with the spectral irradiance responsivities $R_{1}(\lambda)$ and $R_{2}(\lambda)$ of the dual-photodiode radiometer. We confirm that the spectra of all the UV sources used have the finite spectral bandwidth within the operating wavelength range of the dual-photodiode radiometer.

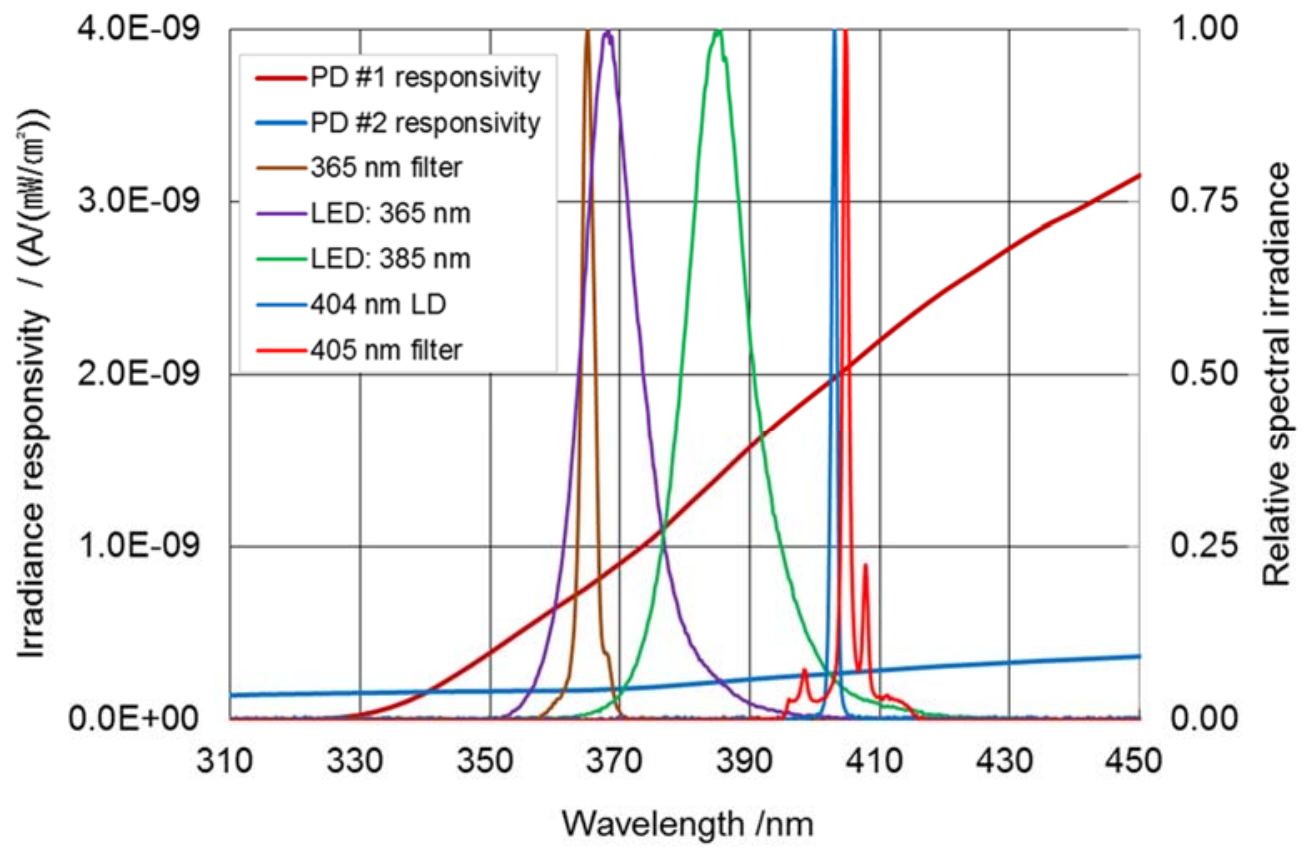

\section{Figure 5 - Spectral distribution of the UV sources used for testing the dual-photodiode reference radiometer for UV-meter calibration.}

\subsection{Centroid wavelength measurement}

The centroid wavelength is determined from the photocurrent readings of the dual-photodiode radiometer by using Eq. (4). Table 1 shows the results of centroid wavelength measured by the dual-photodiode radiometer and by the spectroradiometer. For all the UV sources, the difference is less than $0.2 \mathrm{~nm}$. The maximum difference is obtained for the UV LED at $385 \mathrm{~nm}$, which has a relatively broad spectral bandwidth. This confirms that the error of the dualphotodiode radiometer generally increases with the spectral bandwidth due to the increasing deviation from the linear dependence condition of the spectral responsivities [Shin, 2019].

Table 1 - Centroid wavelengths of UV sources measured by the dual-photodiode radiometer and by the spectroradiometer.

\begin{tabular}{|l|c|c|c|c|c|}
\hline \multicolumn{1}{|c|}{ UV-source } & $\begin{array}{c}\text { Filtered Hg } \\
\text { lamp }(365 \mathrm{~nm})\end{array}$ & LED (365 nm) & LED (385 nm) & LD (404 nm) & $\begin{array}{c}\text { Filtered Hg lamp } \\
(365 \mathrm{~nm})\end{array}$ \\
\hline $\begin{array}{l}\text { Centroid wavelength / nm } \\
\text { (Dual-photodiode radiometer) }\end{array}$ & $\mathbf{3 6 4 . 9 7}$ & $\mathbf{3 6 9 . 7 4}$ & $\mathbf{3 8 6 . 3 6}$ & $\mathbf{4 0 3 . 8 9}$ & $\mathbf{4 0 4 . 9 2}$ \\
\hline $\begin{array}{l}\text { Centroid wavelength / } \mathrm{nm} \\
\text { (Spectroradiometer) }\end{array}$ & 365.14 & 369.80 & 386.17 & 403.87 & 404.98 \\
\hline Difference / nm & -0.17 & -0.07 & 0.19 & 0.02 & -0.06 \\
\hline
\end{tabular}

\subsection{Irradiance measurement}

The spectrally integrated irradiance is determined from the photocurrent reading of the photodiode \#2 based on the spectral irradiance responsivity as well as the determined centroid wavelength by using Eq. (5). Table 2 shows the results of irradiance measured by the dualphotodiode radiometer and by the ECPR. The agreement is good with the relative difference of less than $0.5 \%$, which is smaller than the typical uncertainty of the ECPR. 
Table 2 - Spectral irradiances of UV sources measured by the dual-photodiode radiometer and ECPR.

\begin{tabular}{|l|c|c|c|}
\hline UV source & $\begin{array}{c}\text { Irradiance / }\left(\mathrm{mW} / \mathrm{cm}^{2}\right) \\
\text { (Dual-photodiode radiodmeter) }\end{array}$ & $\begin{array}{c}\text { Irradiance / }\left(\mathrm{mW} / \mathrm{cm}^{2}\right) \\
(\text { ECPR) }\end{array}$ & Difference / \% \\
\hline Filtered Hg lamp $(365 \mathrm{~nm})$ & 65.75 & 66.06 & -0.47 \\
\hline LED $(365 \mathrm{~nm})$ & 2.60 & 2.59 & 0.21 \\
\hline LED $(385 \mathrm{~nm})$ & 5.10 & 5.12 & -0.54 \\
\hline Filtered Hg lamp $(405 \mathrm{~nm})$ & 13.61 & 13.68 & -0.50 \\
\hline
\end{tabular}

\section{Conclusion}

We realized a reference radiometer for calibration of the UVA-meters based on the concept of the dual-photodiode radiometer. Once the spectral irradiance responsivities of the two photodiodes are calibrated, the dual-photodiode radiometer can simultaneously measure both the centroid wavelength and the spectrally integrated irradiance of the light source directly from the photocurrent readings of the two photodiodes. Its working principle is based on the condition that the spectral irradiance responsivities of the two photodiodes should be a linear function against wavelength in the operating wavelength range. In addition, the ratio of the two spectral responsivities should be a monotonic function. In experiment, we realized these conditions by using a coloured filter as a beam splitter for a wavelength range from $330 \mathrm{~nm}$ to $450 \mathrm{~nm}$.

The validity of the new reference radiometer is tested at various UV sources, which are typically used for the UVA-meter calibration: a diode laser at $404 \mathrm{~nm}$, a high pressure $\mathrm{Hg}$ lamp filtered at $365 \mathrm{~nm}$ and $405 \mathrm{~nm}$ with a bandwidth of approximately $15 \mathrm{~nm}$, and two UV LEDs with peak wavelengths at $365 \mathrm{~nm}$ and $385 \mathrm{~nm}$. The centroid wavelengths measured by the dualphotodiode radiometer are compared with a spectroradiometer. For all the UV sources tested, the agreement was better than $0.2 \mathrm{~nm}$. For the irradiance measurement, the results are compared with an ECPR. The relative difference of irradiance between the dual-photodiode radiometer and the ECPR was less than $0.5 \%$ for all the UV sources tested, which is smaller than the measurement uncertainty of the ECPR.

In conclusion, the dual-photodiode radiometer is the promising candidate as the reference radiometer for calibration of the UVA-meters. It ideally combines the advantages of a spectrally flat absolute radiometer and a photodiode-based radiometer. Moreover, it can monitor any spectral change of the UV source by measuring the centroid wavelength. An uncertainty analysis of the dual-photodiode radiometer for the practical application is in progress.

\section{References}

CIE 2016. TC 2-47. Characterization and calibration Method of UV Radiometers, Vienna: CIE.

EPPELDAUER, G. P., PODOBEDOV, V. B., HANSSEN, L. M., and COOKSEY, C. C. 2017. LowNEP pyroelectric detectors for calibration of UV and IR sources and detectors. Proceedings of SPIE, Vol. 10378, 1037809-1 - 1037809-13.

Shin, D.-J., Park, S., and Lee, D.-H. 2019. Dual-photodiode Radiometer Design for Simultaneous Measurement of Irradiance and Centroid Wavelength of Light Sources with Finite Spectral Bandwidth, to be published. 